\title{
Tecnologías de vigilancia en las empresas mexicanas para protegerse de la inseguridad
}

\author{
Lucía Carmina Jasso López ${ }^{1}$
}

Recibido: 06/04/2020; Aceptado: 15/06/2020

Cómo citar: Jasso López, L. C. (2020) Tecnologías de vigilancia en las empresas mexicanas para
protegerse de la inseguridad. Revista Hipertextos, 8 (14), pp. $91-110$.
$\begin{aligned} & \text { https://doi.org } / 10.24215 / 23143924 \mathrm{e} 021 \\ & \text { DOI: }\end{aligned}$

Resumen. El presente artículo tiene como objetivo estudiar los efectos de la violencia y la inseguridad en las empresas mexicanas, enfocándose en la inversión en medidas preventivas que consideran las tecnologías de vigilancia -alarmas y cámaras de video- como alternativa para protegerse de la inseguridad. A partir del análisis estadístico de cuatro encuestas de victimización de empresas en México -2012, 2014, 2016 y 2018- a las que se tuvo acceso a través del Laboratorio de Microdatos del Instituto Nacional de Estadística y Geografía (INEGI), se encontró que las empresas han incrementado su inversión en tecnologías de vigilancia en los últimos años y que esta inversión no está únicamente asociada con la victimización directa, sino también es una medida preventiva que se explica a partir de variables como el entorno a nivel local, la percepción de inseguridad, las fallas de la seguridad pública, entre otras.

Palabras clave: empresas, tecnologías de vigilancia, inseguridad, percepción de inseguridad, encuestas de victimización

\section{Surveillance technologies in Mexican companies to protect themselves from insecurity}

Abstract. This article study the effects of violence and insecurity on mexican businesses focusing on investment in preventive measures that consider surveillance technologies -alarms and video cameras-as an alternative to protect insecurity. Based on the statistical analysis of four victimization surveys against businesses in Mexico -2012, 2014, 2016 and 2018- it is found that businesses have increased their investment in surveillance technologies in recent years, and this investment is not only related to direct victimization, is also a preventive measure that is explained by variables such as the environment at the local level, the perception of insecurity, public security failures, among others.

\footnotetext{
${ }^{1}$ Doctora en Políticas Públicas. Investigadora de Tiempo Completo del Instituto de Investigaciones Sociales de la Universidad Nacional Autónoma de México. Email de contacto: carmina.jasso@sociales.unam.mx 
Key words: businesses, surveillance technologies, insecurity, perception of insecurity, victimization surveys

\section{Tecnologias de vigilância em empresas mexicanas para se proteger da insegurança}

Resumo. O presente artigo tem como objetivo estudar os efeitos da violência e da insegurança nas empresas mexicanas, focando na inversão em medidas preventivas que consideram as tecnologias de vigilância - alarmes e câmeras de vídeo - como alternativas para se proteger da insegurança. A partir do análise estatístico de quatro pesquisas de vitimização de empresas no México -2012, 2014, 2016 e 2018 que foram acessadas no Laboratorio de Microdatos del Instituto Nacional de Estadística y Geografía (INEGI), verificou-se que as empresas têm aumentado a inversão em tecnologias de vigilância nos últimos anos e essa inversão não é associada apenas à vitimização direta, sino também é uma medida preventiva que é explicada a partir de variáveis como o ambiente ao nível local, a percepção de insegurança, as falhas da segurança pública, entre outras.

Palavras-chave: empresas, tecnologias de vigilância, insegurança, percepção de insegurança, pesquisas de vitimização. 


\section{Introducción}

La violencia y la inseguridad producen efectos diversos en la sociedad, merman la calidad de vida de las personas, modifican sus rutinas, así como sus actividades cotidianas y generan importantes costos económicos. En México se ha calculado que, como efecto de la inseguridad en los hogares mexicanos, el costo del delito en 2017 ascendió a 299.6 mil millones de pesos que corresponde al 1.65\% del PIB (INEGI, 2018a: 27). Este costo impactó principalmente en pérdidas económicas directas a consecuencia de los delitos, pero también en la inversión en medidas preventivas e incluso en el pago por daños en la salud. Sin embargo, el mayor impacto del crimen es sobre las personas, tanto de manera directa "convirtiéndoles en sus víctimas" como indirectamente "robándoles la posibilidad de construir sociedades libres del temor" (Rojas, 2007: 98).

Además, la violencia y la inseguridad también afectan a las empresas. En 2012, la Confederación Patronal de la República Mexicana (COPARMEX) anunció que más de 160 mil empresas cerraron "por inseguridad" (Excélsior, 2012). Y a finales de 2018, los datos de COPARMEX indicaron que el 58\% de las empresas agremiadas fue víctima de algún delito entre agosto y noviembre de ese año, con el robo de mercancía, dinero y equipo como el ilícito más común (El Heraldo, 2018). Y de acuerdo a la información pública disponible, en 2017 un total de 1.5 millones de unidades económicas en México fueron víctimas del delito, considerando el total en el país, se estima que el crimen afectó al 33.7\% de las empresas a nivel nacional (INEGI, 2018).

En este contexto, el presente artículo tiene como objetivo estudiar los efectos de la violencia y la inseguridad en las empresas mexicanas, los costos de la delincuencia en las unidades económicas, enfatizando en la inversión en medidas preventivas que consideran las tecnologías de vigilancia como alternativa para protegerse de la inseguridad, como las alarmas y cámaras de vigilancia. La fuente principal de análisis es la Encuesta Nacional de Victimización de Empresas (ENVE) que realiza bianualmente el Instituto Nacional de Estadística y Geografía (INEGI) ${ }^{2}$ desde 2012.

La investigación parte de la hipótesis de que las empresas para protegerse de la inseguridad han incrementado su inversión en tecnologías de vigilancia en los últimos años, y que esta inversión no únicamente está asociada con la victimización directa de las empresas, sino que también es una medida preventiva para evitar ser víctimas del delito, que se explica a partir de variables como la inseguridad de las empresas a nivel nacional y local, las perdidas por victimización, la percepción de inseguridad, las capacidades económicas de las empresas, las fallas de la seguridad pública, entre otras.

En concordancia, es relevante enfatizar que la literatura refiere que "sobre los montos que gastan los ciudadanos y las empresas en vigilancia, seguridad y reposición de los daños físicos causados por los delitos la información es fragmentaria” (Rubio, 1998: 3). Además de que destacan "patrones diferenciales, por niveles de ingreso, en cuanto a la tecnología utilizada por

\footnotetext{
2 Organismo público autónomo responsable de normar y coordinar el Sistema Nacional de Información Estadística y Geográfica en México.
} 
los hogares para su seguridad y en cuanto a la efectividad de ese gasto" (Rubio, 1998: 3) que con probabilidad también se reproducen en las empresas mexicanas.

Con el fin de contextualizar el análisis, la primera parte de este texto se enfoca en el estudio del impacto de la violencia y la inseguridad en las empresas y se profundiza en la relación entre la triada: empresas, tecnología y crimen a partir de la revisión de la literatura correspondiente. Posteriormente, se presentan los resultados del análisis de cuatro ediciones de la ENVE con el fin de conocer cómo se ha modificado el objeto de estudio en el tiempo. Asimismo, a través del análisis estadístico descriptivo y bivariado se presentan los resultados del análisis de los microdatos de la ENVE 2018 con los que fue posible profundizar en la relación de la variable dependiente con otras variables que se incluyen en esta encuesta.

\section{El impacto de la violencia y la inseguridad en las empresas}

Desde el siglo XIX la sociología ha estudiado la relación entre los ciclos económicos y la delincuencia (Bressler, 2009: 1). Al respecto, se asume que el vínculo entre crimen, delito ${ }^{3}$ y economía es amplio, lleno de aristas y con interesantes problemas teóricos y empíricos (Ramírez, 2014: 261).

Los marcos explicativos provenientes de la sociología en torno a la economía y la criminología se agrupan en tres: racionalidad criminal, economía política del crimen y privación económica (Ramírez, 2014). Sin embargo, se sostiene que uno de los supuestos más exitosos que explica la relación entre la economía y el crimen, es aquel que la vincula con la hipótesis de desarrollo económico, donde el argumento central postula que "el crecimiento económico estable y de largo plazo genera riqueza material y mejores condiciones de vida que, a la larga, están asociados con bajos índices de criminalidad" (Ramírez, 2014: 273). Bajo esta hipótesis se admite que "los ciclos económicos indican que, durante los tiempos económicos más difíciles, la actividad criminal aumenta", incluso se han encontrado asociaciones entre determinadas condiciones económicas y tipos específicos de delitos, por ejemplo, el fraude durante tiempos de recesión (Bressler, 2009:2).

La violencia ${ }^{4}$ y la inseguridad tienen efectos diferenciados en las empresas. De manera directa, las empresas y quienes ahí laboran son víctimas de delitos como "el robo de tiendas, el vandalismo, la piratería y la falsificación” (Bressler, 2009: 2), la extorsión, las amenazas, el secuestro, etc. Además, existen diversas formas y modalidades en las que éstos ocurren, en algunos casos los cometen quienes forman parte de la empresa y conocen el funcionamiento, o externos que aprovechan la oportunidad para delinquir. Pero en general:

\footnotetext{
3 Para fines de este texto se comprende que existen relación intrínseca entre el crimen y delito. Sin embargo son conceptos diferentes, si bien el crimen se define por la Real Academia Española, como "delito, sea grave o no grave" un delito para configurarse como tal, debe estar tipificado en los códigos penales.

${ }_{4}^{4}$ Desde el enfoque de la Organización Mundial de la Salud se comprende que la violencia es el "El uso deliberado de la fuerza física o el poder, ya sea en grado de amenaza o efectivo, contra uno mismo, otra persona o un grupo o comunidad, que cause o tenga muchas probabilidades de causar lesiones, muerte, daños psicológicos, trastornos del desarrollo o privaciones" (OMS). Ver: Campaña Mundial de Prevención de la Violencia (2012-2020).

Hipertextos, Vol. 8, N 14. Buenos Aires, Julio/Diciembre de $2020 \ll 94$ DOI: https://doi.org/10.24215/23143924e021, https://revistas.unlp.edu.ar/hipertextos
} 
Los delitos contra las empresas producen pérdida de ganancias por una disminución en la productividad, un incremento en los precios y en las primas de seguros, una mayor sensación de inseguridad entre empresarios y empleados, una pérdida de empleos por motivos de recorte de personal, y en ocasiones también implica el cierre definitivo de empresas o establecimientos, así como un recelo por invertir y expandirse (Vilalta, 2018: 502).

En la situación más extrema, el crimen y la violencia pueden llegar a colapsar y terminar con la vida de las empresas. En Estados Unidos, la Cámara de Comercio ha encontrado que en el 30\% de los casos, el crimen es un factor determinarte de los fracasos de las empresas (Bressler, 2009), así también ha ocurrido en México como se refiere en la introducción de este texto.

Bajo este argumento, no es de extrañar que la violencia y la inseguridad tengan un impacto negativo en la inversión y el crecimiento económico de una nación (Correa, 2013: 30). De manera tal, que otro de los efectos más radicales es la "emigración de empresas por razones de seguridad" (Rubio, 1998: 1) como ha ocurrido en Colombia y México durante los periodos con mayores niveles de violencia.

Los altos niveles de violencia e inseguridad afectan la competitividad de los países, porque merman el funcionamiento de la economía y de las empresas de distintas formas. Aumentan los costos de las empresas, disminuyen la producción y la productividad, afectan los ingresos por ventas y presentan un alto costo de oportunidad por los recursos utilizados para combatirla, así como por las inversiones que no se realizan por el mayor riesgo percibido por este problema (Figueroa, et. al., 1997: 68).

Las empresas cambian rutas e incluso abandonan territorios que consideran inseguros, lo que afecta directamente a las comunidades. Tal como se constata, en los testimonios de los habitantes de barrios populares donde "es recurrente la idea de que cuando una zona se torna demasiado insegura los proveedores dejan de abastecer el comercio local" (Rubio, 1998:4).

Asimismo, provocan la cancelación de inversiones y otras actividades e impactan directamente sobre un segundo aspecto de la competitividad de las empresas: su rentabilidad (Soria, 2017: 33). Una empresa victimizada o vulnerable, disminuye su rentabilidad.

En este contexto, la violencia e inseguridad también inciden directamente en la inversión en medidas de seguridad para evitar ser víctimas del delito. Al respecto es pertinente el modelo de Gary Becker que parte de la teoría de la elección racional para explicar que el crimen se reduce al disminuir los beneficios o al incrementar la probabilidad del castigo (Becker, 1968). Las empresas lo que esperan es que, al incrementar ésta posibilidad de castigo a través de las medidas preventivas, disminuya el crimen y la inseguridad que les acecha.

Desde el punto de vista de Bressler (2009) la prevención es el medio más rentable para reducir el impacto de la criminalidad en los negocios, sobre todo considerando que "la efectiva protección de la ciudadanía y de sus bienes favorece la creación de empresas, la inversión y la generación de empleos, elementos indispensables para el progreso del país” (Soria, 2017: 38).

En la lógica de las empresas, a mayor inversión en medidas preventivas, disminuye el riesgo de ser víctima del delito, "en general se supone que existe una racionalidad calculada en las medidas que se implementan, incluso se considera el aspecto de inevitabilidad" (Stuart, 2014: 
243). En consecuencia, se incrementa la adquisición de estos productos y el mercado que produce y promueve estos bienes se ha convertido en un negocio altamente rentable que crece exponencialmente.

Pero la prevención en las empresas no es un asunto exclusivo de éstas y también debería promoverse desde los gobiernos a través de la política pública de contención de la delincuencia que evite "el doble efecto de la criminalidad en las empresas: inhibir su creación y evitar que éstas, una vez creadas, tengan una mayor probabilidad de ser víctimas de la misma" (Soria, 2017: 38).

\section{Empresas, tecnología y crimen}

La triada: empresas, tecnología y crimen confluye de distintas maneras. En las siguientes páginas se abordará desde dos enfoques: de manera sucinta sobre la empresa como víctima del crimen con el uso de tecnología por parte de las organizaciones delictivas, y de manera más amplia respecto a las empresas que con el fin de protegerse de la inseguridad invierten en tecnología, que es el tema central de esta investigación. Si bien, en ambos enfoques la empresa es víctima, en la segunda aproximación tiene un rol preventivo, ya sea para evitar la victimización, o como mecanismo reactivo para no ser revictimizada. De acuerdo con Vilalta (2018: 503) "los académicos han tendido a estudiar más a las empresas en su rol de victimarias".

Respecto a la empresa como víctima del crimen con el uso de tecnología, es importante contextualizar las "nuevas formas" en las que la violencia y la inseguridad las afectan, tal es el caso de "los ataques a la seguridad cibernética" que amenazan a nivel mundial. Los delitos en este tipo "afectan la privacidad, amenazan la estabilidad de las comunicaciones globales y causan pérdidas muy importantes a los consumidores y empresas ligados al comercio electrónico" (Rojas, 2008: 95).

Numéricamente, de acuerdo con la Encuesta Nacional de Delitos Informáticos 67\% de las empresas en Estados Unidos fueron víctimas de al menos un ataque cibernético (Bressler, 2009: 4). La relevancia de estos ataques reside en que se realizan a distancia, sin contacto físico y pueden llegar a impactar económicamente a mayor escala que el delito convencional, por ejemplo, en un fraude detectado en Francia en 2001 el monto fue equivalente a 2,000 delitos contra la propiedad realizados en ese mismo año (Cervini, 2007).

Es innegable que la mayor parte de las empresas están fuertemente vinculadas con la tecnología a través del comercio electrónico, el uso de la banca electrónica y de manera cotidiana realizan actividades y transacciones económicas a través de internet que pueden ser vulneradas por este tipo de ataques a la seguridad cibernética. Para contrarrestar este tipo de amenazas, las instituciones públicas de seguridad han desarrollado áreas específicas para la prevención de crímenes relacionados con el delito cibernético, así como llevan a cabo actividades reactivas para investigar y sancionar a quienes cometen este tipo de delitos.

Asimismo, las empresas frente a la vulnerabilidad que supone este tipo de ataques han implementado mecanismos para evitar la victimización de sus clientes quienes pueden ser engañados y defraudados por organizaciones criminales a través de internet usurpando la identidad de las empresas. Se advierte que las empresas preocupadas por las consecuencias de 
estos fenómenos criminales "han propiciado importantes estudios en esta materia. Sin embargo, los resultados y recomendaciones de esos trabajos no han sido suficientemente divulgados o puestos en práctica" (Solís, 2008: 139).

Desde luego que también las empresas tienen un papel importante como proveedoras de tecnología para protegerse de la inseguridad. En diversos casos, se han vuelto imprescindibles y hay un mercado creciente que concentra a las empresas que proveen tecnología tanto al sector público como privado para protegerse de la inseguridad. Este mercado se hace patente en exposiciones y ferias que concentran anualmente a las empresas que producen y/o distribuyen las innovaciones tecnológicas en la materia.

Por otra parte, se advierte el fenómeno de la "sofisticación criminal" que se manifiesta en las "falencias" que alcanzan a todos los agentes institucionales y privados (Cervini, 2007). Cada vez más organizaciones criminales consumen las distintas formas de innovación tecnológica que ofrecen las empresas en el mercado -legal e ilegal-, para fortalecer sus estructuras y sus operaciones delictivas. En algunos países las capacidades tecnológicas de la delincuencia, son incluso mayores que las de las instituciones públicas de seguridad.

Por otra parte - desde el segundo enfoque- están las empresas que con el fin de protegerse de la inseguridad invierten en tecnología. La lógica bajo la cual se realiza esta inversión, en particular de las tecnologías de vigilancia, es que se considera como "una medida de la cautela y capacidad que se tiene para prevenir un delito. A menor vigilancia, mayor probabilidad de victimización" (Vilalta, 2018: 512).

$\mathrm{Al}$ respecto hay investigaciones que plantean como hipótesis que "en la medida que crece la inseguridad, las empresas tienden a adoptar más medidas preventivas", sin embargo, se ha encontrado que en las "entidades federativas con delincuencia a la baja se observa una correlación alta y positiva en la inversión en medidas preventivas" (Soría, 2017: 29). Lo anterior, permite advertir que las empresas con "menor riesgo" también invierten en medidas de protección como una forma de prevención, es decir, la inversión no se asocia únicamente con la victimización directa, así como tampoco con los contextos de inseguridad registrados a nivel local.

La inversión por parte de las empresas para protegerse de la inseguridad genera costos, lo que a su vez también puede causar efectos en la competitividad considerando que se genera un "círculo vicioso" en el sentido de que "las medidas de protección adoptadas por las empresas, incrementan sus costos, y a mayores costos, menor competitividad, siendo más afectadas las unidades económicas de las entidades federativas que deben tomar más medidas de protección aún y cuando éstas sean más costosas" (Soría, 2017: 29).

En estas circunstancias, "a medida que el costo de la delincuencia escala y recorta las ganancias y beneficios, las empresas deben aumentar las medidas preventivas y desarrollar métodos más sofisticados para detectar el delito" (Bressler, 2009: 11). En respuesta, cada vez hay más empresas y establecimientos mercantiles que deciden implementar medidas preventivas con el uso de alternativas tecnológicas, desde la instalación de puertas automáticas, control de accesos, cámaras y alarmas, hasta la implementación de servicios integrales o sofisticadas formas de control como las tecnologías biométricas, entre otras (INCIBE, 2016). Así no solo hay una 
sofisticación criminal, sino también una sofisticación preventiva que cada vez incluye más y mejores herramientas tecnológicas.

\section{Metodología}

La metodología de investigación es fundamentalmente cuantitativa. Se recurre al análisis de fuentes secundarias, particularmente de la ENVE del INEGI que "se enfoca en proporcionar información valiosa para el diseño de políticas públicas por parte de las autoridades competentes, así como para contribuir en el diseño de estrategias en materia de seguridad por parte de las empresas" (INEGI, 2018: 2).

La unidad de estudio son las empresas mexicanas. Al respecto, en la literatura se señala que "las condiciones que generaron el interés por las microempresas y las pequeñas y medianas empresas (mipyme) en América Latina en muchos casos se han agudizado por la presencia de nuevos elementos que han de considerarse en el escenario económico internacional (el desarrollo de nuevas tecnologías, el proceso de internacionalización y otros)" (Zevallos, 2003: 54). Entre estos nuevos elementos está el fenómeno de la inseguridad que afecta a las empresas y el uso de tecnología que implementan para protegerse.

En la encuesta se cuestiona respecto a las medidas de protección, según adopción de estas para protegerse del delito. Particularmente, en cuanto a las tecnologías de vigilancia se les pregunta: Para protegerse de la delincuencia, ¿en el establecimiento se realizó algún tipo de medida como instalar alarmas y/o videocámaras de vigilancia?

La ENVE se ha realizado en cuatro ocasiones desde 2012, por lo que se analiza cómo ha variado en el tiempo la inversión en alarmas y/o video cámaras de vigilancia como medida de protección de las empresas. Asimismo, con el fin de profundizar en el estudio se analizan los microdatos $^{5}$ de la edición 2018 de la ENVE cuyo tamaño de la muestra nacional fue de 32,588 unidades económicas. Se realizó análisis estadístico descriptivo y bivariado de la variable dependiente y otras variables de interés con el uso del Paquete Estadístico para las Ciencias Sociales SPSS (por sus siglas en inglés).

\section{Resultados}

En cifras, el impacto de la inseguridad en las empresas mexicanas se condensa en los principales resultados de la ENVE 2018 que reporta datos de 2017 que advierten que 1.5 millones de unidades económicas fueron víctimas del delito (INEGI, 2018: 2).

Respecto a la encuesta realizada en 2015 se registró una ligera disminución de víctimas, al pasar de 3,548 por cada 10,000 unidades económicas en 2015 a 3,373 en 2017 lo que representa un cambio porcentual a la baja de $4.9 \%$. Sin embargo, en 6 entidades se observa un incremento en la tasa de victimización destacando el estado de Durango donde aumentó en 29.6\%, mientras

\footnotetext{
${ }^{5}$ A través del Laboratorio de Microdatos del INEGI se tuvo acceso a la base de datos completa de la ENVE 2018, donde siempre se privilegió el derecho a la protección de datos de los informantes.
}

Hipertextos, Vol. 8, N 14. Buenos Aires, Julio/Diciembre de 2020 «98 DOI: https://doi.org/10.24215/23143924e021, https://revistas.unlp.edu.ar/hipertextos 
que en 21 entidades se mantuvo sin cambio estadísticamente significativo y en 5 demarcaciones el cambio fue a la baja.

Entre las empresas que fueron víctimas del delito, las más afectadas son las empresas del sector comercio (38.4\%) seguido del sector industria $(30.6 \%)$ y el sector servicios $(29.3 \%)$. Afectó en mayor medida a las medianas empresas $(61.5 \%)$ y en menor medida a las microempresas (32.9\%) (INEGI, 2018: 2).

Los delitos de los que mayormente son víctimas las empresas mexicanas son: el robo hormiga, robo o asalto de mercancía, dinero, insumos o bienes y extorsión. En promedio, cada empresa que fue víctima del delito, tuvo una tasa de concentración de 2.5 delitos (INEGI, 2018: 2).

Como se sostiene en la primera parte de este texto, la violencia y la inseguridad tienen efectos directos en las empresas. En la ENVE 2018 hay evidencia de que las empresas manifiestan sentirse inseguras: el 56.2\% respecto a transportar productos por carreteras o autopistas, $55.7 \%$ para movilizar productos en corredores industriales, 53\% hacer compraventa en centrales de abasto, 48.8\% hacer transacciones en bancos y 38.2\% realizar compraventa en centros comerciales. Por otro lado, en la ENVE 2016 se encuentra que la percepción de inseguridad incrementó sensiblemente respecto a las actividades mencionadas.

Los delitos perpetrados contra las empresas en 2017 representan un monto de 155.8 mil millones de pesos, que equivalen al $0.86 \%$ del PIB. Este monto es superior al registrado en 2015 que alcanzó los 153.3 mil millones de pesos. En este sentido, es pertinente señalar que, aunque se registró un decremento en el porcentaje de empresas que fueron víctimas, el impacto económico en 2017 fue mayor.

El costo promedio del delito por unidad económica a nivel nacional en 2017 fue de 66,031 pesos, monto superior al registrado en 2015 que fue de 57,779 pesos. En entidades como Coahuila y Ciudad de México el costo promedio por unidad económica es superior a los 100,000 pesos y en Nayarit, entidad con el costo promedio más bajo a nivel nacional, es de 26,325 pesos.

A este monto se suma el gasto en medidas preventivas que en 2017 fue de 44.1 mil millones de pesos a nivel nacional. En 2017 de cada 10 empresas, 4 invirtieron en alguna forma de protección. En relación con esta inversión, estudios previos han mostrado que "hay una clara geografía de la protección contra el delito en términos del gasto promedio dirigido a la adquisición de medidas de protección", además de que "sí se correlaciona en el tiempo" (Vilalta, 2017: 849).

En primer lugar, se encuentra la inversión para "cambiar o colocar cerraduras o candados" con $28.7 \%$, seguido de "instalar alarmas y/o videocámaras de vigilancia" con 16.2\%. Mientras que $5.9 \%$ de las empresas optó por "contratar o mantener vigilancia y seguridad privada." 6 Se trata de un porcentaje relativamente bajo, debido a que "la contratación de vigilancia privada es muy onerosa y no accesible para la mayor parte de las empresas del país” (Vilalta, 2017: 847).

\footnotetext{
${ }^{6}$ Se refiere que sobre "los servicios de vigilancia privada, la información es limitada. Existen datos sobre el personal dedicado a esa labor, pero únicamente en las empresas legales y reguladas” (Rubio, 1998:3).

Hipertextos, Vol. 8, N 14. Buenos Aires, Julio/Diciembre de 2020 «99 DOI: https://doi.org/10.24215/23143924e021, https://revistas.unlp.edu.ar/hipertextos
} 


\subsection{Tecnologías de vigilancia en las empresas}

De acuerdo con las estimaciones del INEGI, -en promedio desde 2012 hasta 2018-, el 14.2\% de las empresas han optado por las tecnologías de vigilancia como medida de protección, lo que corresponde a la instalación de aproximadamente 2, 244,734 alarmas y/o video cámaras de vigilancia. $^{7}$

En el tiempo se observa que el porcentaje de empresas que invierten en este tipo de medidas ha incrementado, al pasar de 464,255 empresas (13.4\%) en 2012 a 698,982 empresas (16.2\%) en 2018 (Ver Tabla 1). Esto representa un aumento del 24.3\% en los últimos 6 años.

Tabla $\mathbf{n}^{\mathbf{0}}$ 1. Unidades económicas que instalaron alarmas y/o video cámaras de vigilancia como medida de protección para protegerse del delito (2012-2018)

\begin{tabular}{|c|r|r|r|}
\hline \multirow{2}{*}{ Edición } & \multirow{2}{*}{$\begin{array}{c}\text { Total de } \\
\text { unidades }\end{array}$} & \multicolumn{2}{|c|}{$\begin{array}{c}\text { Instalaron alarmas y/o video } \\
\text { cámaras de vigilancia }\end{array}$} \\
\cline { 3 - 4 } & $3,461,838$ & Absolutos & \multicolumn{1}{c|}{ Relativos } \\
\hline 2012 & $3,629,665$ & 464,255 & 13.4 \\
\hline 2014 & $4,317,248$ & 469,375 & 12.9 \\
\hline 2016 & $4,304,251$ & 612,123 & 14.2 \\
\hline 2018 & 698,982 & 16.2 \\
\hline
\end{tabular}

Fuente: elaboración propia con datos del INEGI, ENVE 2012-2018

Particularmente destaca que las tecnologías de vigilancia como medida para protegerse del delito se encuentran entre las primeras opciones de las empresas, a diferencia de los hogares donde este tipo de medidas a nivel nacional se ubica entre las últimas alternativas de preferencia (Jasso, 2019).

En los años en los que se ha realizado la ENVE se observa que a nivel nacional durante 2012 y 2014 las tecnologías de vigilancia ocuparon el tercer lugar de preferencia, mientras que en los años 2016 y 2018 pasó al segundo lugar. Lo mismo ocurrió en la mayoría de las entidades federativas, de manera tal que, en 2012 en 6 entidades (18.8\%) ocupó el segundo lugar de preferencia, 9 (28.1\%) en 2014, 19 (59.4\%) en 2016, y en 2018 un total de 23 entidades $(71.9 \%)$ refirieron esta medida como la segunda preferencia (Ver Tabla 2). Lo anterior muestra que en el tiempo la inversión en tecnologías de vigilancia para protegerse del delito se ha ido posicionando entre las alternativas preferidas de las empresas. De esta forma sobresale que, en Chihuahua durante 2018, se convirtió en la primera medida de preferencia de las empresas.

En la ENVE 2018 el promedio nacional de empresas que invirtieron en alarmas y videovigilancia es de $16.2 \%$, no obstante, en 15 entidades se registra el mismo porcentaje o uno

\footnotetext{
7 En la mayoría de los casos, las empresas deciden instalar cuando menos dos cámaras como parte de su estrategia de control y seguridad, por lo que esta cifra podría duplicarse o triplicarse y alcanzar más de 5 millones de cámaras de videovigilancia en las empresas mexicanas.
}

Hipertextos, Vol. 8, N 14. Buenos Aires, Julio/Diciembre de 2020 «100 DOI: https://doi.org/10.24215/23143924e021, https://revistas.unlp.edu.ar/hipertextos 
superior. Destacan Querétaro con 22.6\%, Ciudad de México con 21.8\%, Baja California con $20.7 \%$, Aguascalientes con $20.4 \%$ y Chihuahua con 20.1\% (Ver Tabla 2). Lo que significa que en estas entidades 1 de cada 5 empresas optan por este tipo de medidas de protección. ${ }^{8}$ En contraparte Chiapas, Nayarit y Guerrero son las entidades en donde las empresas invirtieron en menor proporción en este tipo de medidas de protección, con 10.8\%, 10.3\% y 9.0\% respectivamente.

En cuanto al tamaño de la empresa, la evidencia muestra que "las empresas pequeñas pueden ser particularmente vulnerables a la delincuencia, ya que las pequeñas empresas a menudo no disponen de medidas de seguridad" (Bressler, 2009: 2). En los resultados de la ENVE se observa que la percepción de inseguridad incrementa en relación al tamaño de la empresa, de tal manera que $31.1 \%$ de las empresas grandes declaran sentirse inseguras, mientras este porcentaje incrementa hasta $51 \%$ en las microempresas. Además, en general se observa que las empresas mexicanas invierten en alarmas y cámaras de videovigilancia, sin embargo, esta proclividad tiende a ser mayor al aumentar el tamaño de la empresa. Así, mientras en promedio dos de cada diez micro y pequeñas empresas invierten en este tipo de medidas, cuatro de cada diez medianas y grandes empresas lo realizan.

Tabla $\mathbf{n}^{\mathbf{o}} 2$. Unidades económicas por entidad federativa que instalaron alarmas y/o video cámaras de vigilancia como medida de protección para protegerse del delito (2012-2018)

\begin{tabular}{|c|c|c|c|c|c|c|c|c|c|c|c|c|}
\hline & \multicolumn{3}{|c|}{ ENVE 2012} & \multicolumn{3}{|c|}{ ENVE 2014} & \multicolumn{3}{|c|}{ ENVE 2016} & \multicolumn{3}{|c|}{ ENVE 2018} \\
\hline & Absoluto & Relativo & $\begin{array}{l}\text { Posición } \\
\end{array}$ & Absoluto & Relativo & $\begin{array}{l}\text { Posición } \\
\end{array}$ & Absoluto & $\begin{array}{l}\text { Relativo } \\
\end{array}$ & Posición & Absoluto & \begin{tabular}{|l|} 
Relativo \\
\end{tabular} & Posición \\
\hline Agrascalientes & 6,599 & 15.9 & 2 & 6,668 & 15.4 & 2 & 10,357 & 20.4 & 2 & 10,543 & 20.4 & 2 \\
\hline Baja California & 12,958 & 15.7 & 4 & 16,460 & 19.7 & 2 & 18,127 & 17.9 & 2 & 21,712 & 20.7 & 2 \\
\hline Campeche & 3,162 & 12.9 & 4 & 3,071 & 11.3 & 4 & 3,928 & 11.7 & 4 & 5,903 & 17.3 & 3 \\
\hline Coahuila & 11,612 & 14.8 & 3 & 12,762 & 15.6 & 3 & 19,090 & 21.0 & 3 & 13,232 & 15.8 & 2 \\
\hline Colima & 3,238 & 12.6 & 2 & 3,383 & 12.5 & 3 & 3,858 & 12.4 & 2 & 4,856 & 15.7 & 2 \\
\hline Chiapas & 9,047 & 7.4 & 4 & 13,138 & 10.8 & 5 & 11,297 & 7.0 & 5 & 17,405 & 10.8 & 4 \\
\hline Durango & 6,320 & 14.3 & 3 & 5,259 & 11.6 & 3 & 4,557 & 9.3 & 3 & 6,009 & 11.0 & 2 \\
\hline Guanajuato & 24,968 & 14.0 & 4 & 32,748 & 18.2 & 2 & 34,980 & 15.6 & 4 & 43,072 & 18.5 & 2 \\
\hline Guerrero & 6,063 & 6.7 & 4 & 6,376 & 6.5 & 4 & 7,604 & 5.9 & 3 & 11,422 & 9.0 & 3 \\
\hline Hidalgo & 7,643 & 9.6 & 4 & 8,490 & 10.9 & 4 & 17,109 & 17.1 & 2 & 13,565 & 13.5 & 2 \\
\hline Jalisco & 33,177 & 12.6 & 2 & 36,314 & 13.3 & 2 & 40,237 & 12.4 & 2 & 64,137 & 19.5 & 2 \\
\hline México & 44,354 & 9.8 & 4 & 50,086 & 10.9 & 4 & 75,072 & 14.0 & 2 & 74,474 & 14.2 & 2 \\
\hline Michoacán & 15,801 & 10.7 & 3 & 19,030 & 11.0 & 3 & 31,246 & 15.4 & 2 & 32,940 & 16.6 & 2 \\
\hline Querétaro & 8,926 & 15.5 & 2 & 8,340 & 14.2 & 2 & 14,382 & 19.1 & 2 & 16,890 & 22.6 & 2 \\
\hline Quintana Roo & 7,393 & 18.4 & 3 & 5,291 & 14.4 & 3 & 10,828 & 21.1 & 2 & 9,653 & 18.6 & 3 \\
\hline San Luis Potosí & 11,596 & 15.2 & 4 & 9,458 & 11.8 & 3 & 11,320 & 12.0 & 2 & 14,411 & 15.7 & 2 \\
\hline Sinaloa & 7,389 & 11.2 & 4 & 9,063 & 12.0 & 3 & 9,657 & 10.1 & 2 & 10,228 & 11.5 & 2 \\
\hline Sonora & 11,154 & 14.2 & 3 & 8,423 & 10.1 & 4 & 16,727 & 17.9 & 3 & 17,273 & 18.0 & 3 \\
\hline Tabasco & 3,642 & 7.5 & 4 & 6,491 & 13.4 & 2 & 9,855 & 16.5 & 3 & 9,824 & 16.2 & 3 \\
\hline Tamaulipas & 14,902 & 15.0 & 3 & 12,772 & 13.0 & 4 & 15,198 & 14.0 & 4 & 13,976 & 12.8 & 4 \\
\hline Tlascala & 4,257 & 8.8 & 4 & 6,056 & 12.0 & 3 & 8,236 & 13.2 & 2 & 10,642 & 16.1 & 2 \\
\hline Veracruz & 16,765 & 8.5 & 4 & 20,936 & 10.0 & 4 & 22,966 & 9.7 & 4 & 32,793 & 13.9 & 2 \\
\hline Yucatán & 5,894 & 9.0 & 4 & 7,129 & 9.7 & 3 & 11,084 & 11.8 & 3 & 12,452 & 12.4 & 2 \\
\hline Zacatecas & 3,703 & 7.8 & 3 & 4,356 & 9.0 & 3 & 6,638 & 12.3 & 2 & 8,703 & 15.5 & 2 \\
\hline
\end{tabular}

Fuente: elaboración propia con datos del INEGI, ENVE 2012, 2014, 2016 y 2018. La posición se refiere al lugar de preferencia en cada entidad.

\footnotetext{
${ }^{8}$ En suma, considerando el total de unidades económicas, estas cinco entidades conjuntan 158,741 empresas lo que representa el $22.7 \%$ del total a nivel nacional.
}

Hipertextos, Vol. 8, N 14. Buenos Aires, Julio/Diciembre de 2020 «101 DOI: https://doi.org/10.24215/23143924e021, https://revistas.unlp.edu.ar/hipertextos 


\subsection{Tecnologías de vigilancia, percepción de inseguridad y miedo al delito en las empresas}

Del total de las empresas entrevistadas, el $50.6 \%$ se percibe inseguro en la colonia o localidad en la que se ubican y ofrecen sus bienes o servicios, $70.6 \%$ en el municipio o alcaldía y $78.9 \%$ en la entidad federativa. Es decir, en correspondencia con los resultados de la encuesta que se realiza en los hogares, la percepción de inseguridad incrementa en los lugares más distantes al lugar de residencia de las personas (Jasso, 2019).

En el caso de las alarmas y cámaras de videovigilancia es mayor la inversión en este tipo de tecnologías en las empresas que refieren sentirse inseguras. No obstante, no se advierte esta relación respecto a los distintos lugares, y el promedio de empresas que las instalaron es similar, aunque ligeramente mayor en el caso de las empresas que se sienten inseguras en la colonia o localidad.

También se encuentra mayor propensión a invertir en tecnología cuando considerando el nivel de delincuencia a futuro en la colonia, municipio o entidad federativa, se estima que las condiciones de inseguridad seguirán igual o empeorarán. Es decir, se invierte en esta forma de protección como una manera de prevención ante la prospectiva situacional de inseguridad.

A partir de la teoría del lugar peligroso que refiere que "son las características del lugar, no las de la persona o la empresa en este caso, lo que explica su victimización" (Vilalta, 2018: 511), es relevante conocer las percepciones respecto a los factores situacionales y contextuales en donde se sitúan los establecimientos.

En la encuesta se consulta a los informantes de las empresas: ¿Sabe usted o ha escuchado si en los alrededores del establecimiento suceden o se dan las siguientes situaciones? Con lo que es posible profundizar en las percepciones de inseguridad y las distintas situaciones o factores de riesgo que afirman los informantes existen alrededor de sus ámbitos de acción más próximos.

Lo primero a observar es que en la mayoría de los casos -excepto "venta ilegal de alcohol"hay una mayor prevalencia a invertir en alarmas y cámaras de videovigilancia cuando se advierte la ocurrencia de la situación o factor de riesgo en los entornos. Sobresale que las situaciones que afectan directamente a las empresas, como el delito de extorsión son las que en mayor medida se relacionan con la propensión a invertir en alarmas y cámaras. Tal es el caso de la extorsión de autoridades contra establecimientos, el cobro de piso por delincuentes y las extorsiones por delincuentes (Ver Tabla 3). Sin embargo, como advierte Vilalta (2017) este tipo de medidas difícilmente podrían proteger a las empresas de la mayor parte de las extorsiones.

Hipertextos, Vol. 8, N 14. Buenos Aires, Julio/Diciembre de 2020 «102 DOI: https://doi.org/10.24215/23143924e021, https://revistas.unlp.edu.ar/hipertextos 
Tabla $\mathbf{n}^{\mathbf{0}}$ 3. Empresas que han instalado alarmas y cámaras de videovigilancia y su conocimiento sobre situaciones que ocurren en los alrededores del establecimiento 2018

\begin{tabular}{|l|c|c|}
\hline \multirow{2}{*}{\multicolumn{1}{|c|}{ Situación }} & \multicolumn{2}{c|}{ Sabe o ha escuchado } \\
\cline { 2 - 3 } & Sí & No \\
\hline 1. Se consume alcohol en la calle & $26.4 \%$ & $25.8 \%$ \\
\hline 2. Existe pandillerismo o bandas violentas & $30.3 \%$ & $23.7 \%$ \\
\hline 3. Hay vandalismo contra establecimientos & $32.2 \%$ & $22.9 \%$ \\
\hline 4. Existe venta ilegal de alcohol & $23.9 \%$ & $26.5 \%$ \\
\hline 5. Hay invasión de predios & $30.8 \%$ & $25.2 \%$ \\
\hline 6. Se consume droga & $28.5 \%$ & $24.1 \%$ \\
\hline 7. Existen robos o asaltos frecuentes a establecimientos & $32 \%$ & $20.1 \%$ \\
\hline 8. Se vende droga & $28 \%$ & $25.4 \%$ \\
\hline 9. Existe prostitución & $28.6 \%$ & $25.5 \%$ \\
\hline 10. Hay secuestros & $31.9 \%$ & $24.7 \%$ \\
\hline 11. Hay homicidios & $31.3 \%$ & $24.2 \%$ \\
\hline 12. Hay extorsiones por delincuentes & $36.1 \%$ & $22.4 \%$ \\
\hline 13. Existe cobro de piso por delincuentes & 34.8 & $24.8 \%$ \\
\hline 14. Hay extorsión de autoridades contra establecimientos & 36.5 & $25.1 \%$ \\
\hline 15. Otra situación & 37.5 & $26 \%$ \\
\hline
\end{tabular}

Fuente: elaboración propia con análisis de los microdatos de la ENVE 2018. En la tabla no se contabiliza la respuesta "No sabe" o "No contestó". Los porcentajes corresponden a las empresas que decidieron invertir en videovigilancia $(\mathrm{N}=7,297)$.

Posteriormente se ubican aquellas situaciones que vulneran directamente el funcionamiento del establecimiento como el vandalismo y los robos o asaltos frecuentes a establecimientos. Así también, se colocan las situaciones que atentan contra la seguridad de las personas en las empresas como los secuestros y los homicidios, ambos delitos de alto impacto que transgreden la libertad y la vida.

Finalmente, se encuentran las potenciales violencias e inseguridades que ocurren en el entorno de los establecimientos como, pandillerismo o bandas violentas, prostitución, consumo o venta de droga y consumo de alcohol en la calle.

Respecto a estas conductas denominadas delictivas o antisociales, se observa que la conducta de mayor ocurrencia de acuerdo con las estimaciones de la ENVE, es el consumo de alcohol $(56.3 \%)$, mientras que en menor medida se encuentra el cobro de piso por delincuentes $(14.8 \%)$ y la extorsión por parte de autoridades (10.3\%). Sin embargo, como se ha presentado previamente, estos últimos delitos son los que más se relacionan con la prevalencia de alarmas y cámaras de vigilancia en las empresas y en menor medida el consumo de alcohol.

Lo anterior significa que la conducta delictiva o antisocial que se afirma ocurre con mayor frecuencia en los alrededores, no es la que más incide en la adquisición de tecnologías de vigilancia para protegerse del crimen, sino que son aquellos delitos que, aunque se afirme ocurren con menor frecuencia, afectan en mayor medida al patrimonio e integridad de las empresas y de quienes ahí laboran. 
Para medir el miedo al delito ${ }^{9}$ en las empresas y la propensión a invertir en alarmas y cámaras de videovigilancia, en la ENVE se incorpora la siguiente pregunta: Considerando el nivel de delincuencia, ¿cree que en lo que resta de 2018 el establecimiento pueda ser víctima de...? Y se enlistan 4 distintos delitos.

Sobresale en general, que a mayor miedo al delito las empresas tienen mayor proclividad a invertir en tecnologías de vigilancia para protegerse de la violencia e inseguridad. En mayor nivel están los delitos relacionados con los vehículos como el robo total de las unidades y de las mercancías transportadas y después se ubican los delitos contra el establecimiento y las personas que ahí laboran como el robo o asalto y la extorsión (Ver Tabla 4).

Tabla $\mathbf{n}^{\mathbf{0}}$ 4. Empresas que han instalado alarmas y cámaras de videovigilancia y miedo al delito 2018

\begin{tabular}{|l|c|c|}
\hline \multirow{2}{*}{ Delito } & \multicolumn{2}{c|}{ Miedo a ser víctima del delito } \\
\cline { 2 - 3 } & Sí & No \\
\hline $\begin{array}{l}\text { Robo total de vehículo (automóvil, camioneta, camión o tráiler) } \\
\text { propiedad del establecimiento }\end{array}$ & $42 \%$ & $25.1 \%$ \\
\hline Robo o asalto en sus instalaciones (excluye vehículos) & $32 \%$ & $22 \%$ \\
\hline Robo de mercancías transportada en vehículos & $40.9 \%$ & $22.7 \%$ \\
\hline Extorsión & $33.6 \%$ & $22.1 \%$ \\
\hline Otro acto que afecte su seguridad & $52.4 \%$ & $25.8 \%$ \\
\hline
\end{tabular}

Fuente: elaboración propia con análisis de los microdatos de la ENVE 2018. En la tabla no se contabiliza la respuesta "No sabe" o "No contestó". Los porcentajes corresponden a las empresas que decidieron invertir en videovigilancia ( $\mathrm{N}=7,297)$

De igual manera a como ocurrió en caso de las conductas denominadas delictivas o antisociales, el tipo de delito al que se tiene mayor temor -el $50.4 \%$ de las empresas se percibe como posible víctima de robo o asalto en sus instalaciones- no es el delito que mayor relación tiene con la instalación de alarmas y cámaras de videovigilancia.

Respecto a la victimización, al realizar un análisis de evaluación de los últimos doce meses, se observa que la mayor parte de las empresas consideran que los delitos que las afectan de forma directa se incrementaron, y justamente éstas son las que mayor inversión realizan en tecnología para protegerse de la inseguridad. Probablemente como una forma de prevención.

\subsection{Tecnologías de vigilancia y otras formas de prevención en las empresas}

Regularmente, con el fin de evitar ser vulnerables a la violencia e inseguridad, las empresas implementan distintas medidas de seguridad al interior y exterior de sus establecimientos. Como lo explica Bressler (2009: 6) "la prevención del robo en las tiendas requiere cámaras de vigilancia, espejos y guardias de seguridad", así como otras diferentes medidas que podrían considerarse

9 En el que se "establece una relación directa con algún tipo de delito o el cálculo o posibilidad de ser víctima del delito" (Jasso, 2013).

Hipertextos, Vol. 8, N 14. Buenos Aires, Julio/Diciembre de 2020 «104 DOI: https://doi.org/10.24215/23143924e021, https://revistas.unlp.edu.ar/hipertextos 
complementarias y que "pueden ser de tipos muy variados y no son exclusivas entre ellas" (Vilalta, 2017: 847).

En la ENVE se advierte que las empresas invierten en distintas formas de prevención, encontrándose en los primeros lugares de preferencia las tecnologías de vigilancia. ${ }^{10}$ Lo primero a destacar es que las empresas que invierten en alarmas y videovigilancia tienen una alta probabilidad de instalar otras distintas formas de prevención (Ver Figura 1).

En primer lugar de preferencia -de las empresas que han instalado alarmas y cámaras de videovigilancia- destaca la instalación de dispositivos de localización: GPS, seguida de otras medidas como los sistemas de protección contra ataques cibernéticos; crear un área dentro del establecimiento responsable de la seguridad; comprar cajas o cuartos de seguridad o contratar seguros. En promedio siete de cada diez empresas que han invertido en alarmas y videovigilancia también han adoptado estas medidas para protegerse del delito.

Figura $\mathbf{n}^{\mathbf{0}}$ 1. Empresas que han instalado alarmas y cámaras de videovigilancia y otras medidas de protección para protegerse del delito, 2017

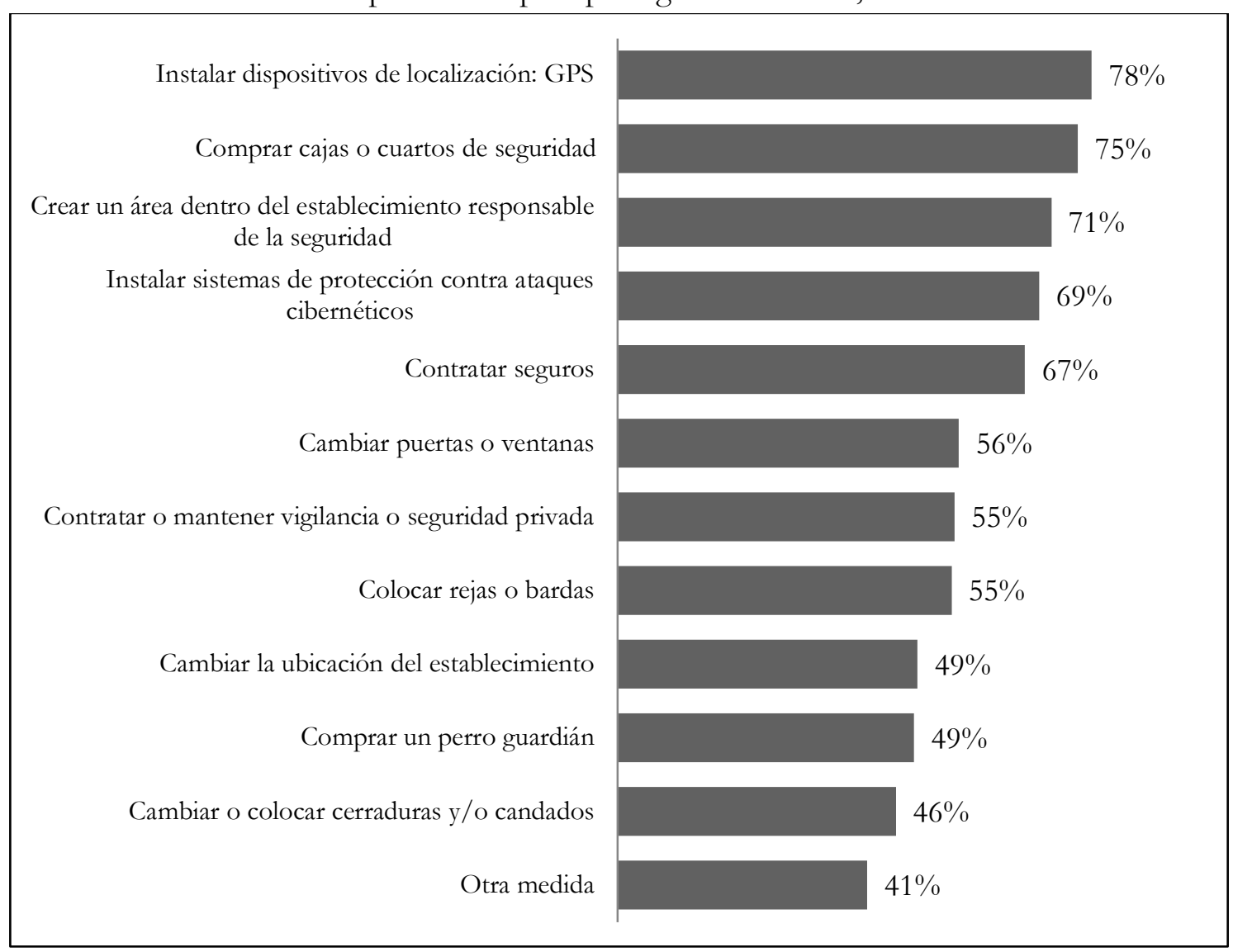

Fuente: elaboración propia con análisis de los microdatos de la ENVE 2018. Los porcentajes corresponden a las empresas que decidieron invertir en videovigilancia $(\mathrm{N}=7,297)$

10 El primer lugar lo ocupa "Cambiar o colocar cerraduras y/o candados" (28.7\%), y el segundo lugar se ubica "Instalar alarmas y/o video cámaras de vigilancia" (16.2\%). 
Entre las medidas menos populares que acompañan la instalación de alarmas y cámaras de videovigilancia se encuentra cambiar o colocar cerraduras y/o candados y comprar un perro guardián. En promedio cuatro de cada diez empresas también han optado por estas formas de prevención.

Tomando en consideración la percepción de inseguridad, el miedo al delito y la victimización de las empresas es importante referir que desde la teoría de las actividades rutinarias:

se pueden analizar las variaciones en los niveles de convergencia de los factores para la comisión de los delitos según las horas de operación de los establecimientos, el uso de videocámaras u otros mecanismos de seguridad, el control de entradas y salidas en almacenes, los protocolos de seguridad de los vehículos y transporte de las mercancías, entre otras medidas preventivas situacionales" (Vilalta, 2018: 509).

Probablemente cada establecimiento cuenta con un análisis propio a partir del cual puede mejorar el funcionamiento de sus medidas preventivas y hacer más eficiente la prevención del crimen. Al respecto, en la ENVE se incluye información relevante desde la teoría de las actividades rutinarias, tales como el tipo de delitos de los que con mayor frecuencia son víctimas las empresas, los modus operandi, los costos del delito, los efectos, entre otros.

\subsection{Tecnologías de vigilancia en las empresas y fallas de la seguridad pública}

Existen distintos tipos de tecnologías de vigilancia, y hasta aquí se ha abordado la que se instala con recursos privados de las empresas, pero también hay videovigilancia pública de los gobiernos locales que podría beneficiar directamente a los establecimientos. En la ENVE es posible explorar el conocimiento que tienen los informantes de las empresas respecto a esta videovigilancia pública, de esta forma en el módulo correspondiente al Desempeño Institucional se les consulta: ¿ ¿Sabe usted si las autoridades realizaron alguna de las siguientes acciones en el 2017 en su (municipio/delegación) para mejorar la seguridad, como.... la instalación de videocámaras de vigilancia?

Bajo esta lógica, la hipótesis a probar consiste en que a menor conocimiento de la instalación de cámaras públicas, incrementaría la videovigilancia privada, es decir que "habría una sustitución de seguridad pública por seguridad privada por parte de las mismas empresas" (Vilalta, 2017: 853), donde también debiera considerarse además del conocimiento, la percepción que tienen respecto a esta videovigilancia pública.

En promedio, a nivel nacional el $18.3 \%$ de las empresas afirmó tener conocimiento de la instalación de videocámaras de vigilancia públicas, ${ }^{11}$ pero en Ciudad de México, ${ }^{12}$ Michoacán de

11 Este porcentaje disminuyó de 22.4\% en 2016 a 18.3\% en 2018. En 2018 la acción que se refiere con mayor frecuencia por parte de los entrevistados es el mejoramiento del alumbrado en los alrededores del establecimiento (INEGI, 2018a).

${ }^{12}$ En el caso de la Ciudad de México, el alto nivel de conocimiento por parte de los informantes coincide con que justamente esta demarcación es la que tiene mayor número de cámaras públicas instaladas a nivel nacional. El C5 de la Ciudad de México a julio de 2020 opera y monitorea más de 15 mil cámaras de vigilancia.

Hipertextos, Vol. 8, N 14. Buenos Aires, Julio/Diciembre de 2020 «106 DOI: https://doi.org/10.24215/23143924e021, https://revistas.unlp.edu.ar/hipertextos 
Ocampo, Yucatán y Campeche más del 30\% afirmaron tener conocimiento de este tipo de medidas por parte de las autoridades. En contraparte, en 8 entidades federativas (Baja California, San Luis Potosí, Durango, Veracruz de Ignacio de la Llave, Colima, Oaxaca, Tabasco y Nayarit) el conocimiento de esta videovigilancia pública es menor al 10\%, es decir, solo una pequeña parte de las empresas.

Al hacer este análisis con los resultados estatales difundidos en los tabulados del INEGI, se encontró una correlación positiva muy baja $\left(\mathrm{R}^{2}=0.028\right)$ es decir, prácticamente no existe asociación entre el conocimiento de la videovigilancia pública y la inversión en vigilancia privada. Se observa que aún y cuando se refiere conocimiento de la videovigilancia pública el $5.6 \%$ de las empresas invierte en su propia videovigilancia, porcentaje muy similar a las empresas que refieren no tener conocimiento. Sin embargo, en el caso de quienes afirman que este tipo de acciones no se realizó por parte de las autoridades, la inversión privada es de 15\%, esto significa, es superior. Estos resultados muestran que se trata en gran medida de "un costo producto de los fallos en materia de seguridad pública" y que "la seguridad privada tiene auge donde la seguridad pública falla" (Vilalta, 2017: 863).

Así, podría advertirse que la videovigilancia privada es predominantemente una inversión ante la ausencia de videovigilancia pública por parte de las autoridades locales. Aunque también se trata de una inversión complementaria que en algunos casos funciona en esquemas de colaboración con los centros de monitoreo público.

Por ejemplo, en la Ciudad de México en agosto de 2019 se anunció que "empresas y asociaciones tendrán posiciones en el $\mathrm{C} 5$, donde podrán recibir alertas y atender casi inmediatamente una incidencia" (Cosme, 2019). Si bien, esto tiene un costo para los establecimientos, representa la oportunidad de tener una comunicación directa con los centros de monitoreo públicos y en esta medida poder recibir atención policial o de los distintos tipos de atención de emergencias en caso de ser necesario.

De forma similar ocurre en el C5 del Estado de México en donde asociaciones de empresarios tienen contacto directo con el centro de monitoreo en caso de alguna emergencia ${ }^{13}$ e incluso han desarrollado el llamado "Código C5" con el cual, a través de un botón de pánico que se dispone de forma gratuita para los afiliados, se tiene respuesta inmediata del C5 para atender emergencias (Nader, 2019).

Asimismo, en varias entidades federativas se ha anunciado la conexión directa de las cámaras privadas de los comercios y negocios a los centros de monitoreo público, de tal manera que, al reportar una emergencia esta sea de conocimiento inmediato de las autoridades quienes podrán acceder a las cámaras para visualizar la emergencia.

La relevancia de este tipo de acciones -en la que las cámaras privadas se enlazan con las plataformas gubernamentales- es de tal magnitud que incluso se habla de la "videovigilancia colaborativa" (Orquiz, 2019). Además dejan claro "el valor de la colaboración entre los sectores público y privado en la prevención de la delincuencia y en la reducción de pérdidas" en las empresas (Stuart, 2014: 246).

${ }^{13}$ En la audiencia pública que se transmitió en vivo y a la cual asistí el 25 de febrero de 2020, varios representantes de empresarios afirmaron que reciben atención directa a través del Código C5.

Hipertextos, Vol. 8, N 14. Buenos Aires, Julio/Diciembre de 2020 «107 DOI: https://doi.org/10.24215/23143924e021, https://revistas.unlp.edu.ar/hipertextos 


\section{Conclusiones}

La violencia y la inseguridad tienen diferentes efectos. En el caso de las empresas genera costos directos e indirectos, donde el más extremo puede llegar a ser el colapso que orilla a cambiar los establecimientos de lugar, modificar rutas o incluso desaparecer. Entre esos costos se encuentra el correspondiente a las medidas para protegerse de la inseguridad.

En México, las tecnologías de vigilancia se han posicionado entre las medidas de mayor preferencia para protegerse del delito. En el tiempo se advierte que esta inversión se ha ido colocando entre las alternativas favoritas de las empresas al pasar del tercer al segundo lugar de preferencia. Además, en el periodo analizado es claro que a nivel nacional se observa un incremento en el porcentaje de empresas que optan por estas tecnologías y las adquieren e instalan con sus propios recursos. Estamos, como se ha estudiado en otras latitudes, en "la era de la videovigilancia” (Sanllehí, 2009).

Los hallazgos a partir del análisis de la ENVE permiten conocer que esta inversión no únicamente está correlacionada con la victimización directa de las empresas, sino que también es una medida de prevención para protegerse de la inseguridad, que se explica de las expectativas relacionadas con la inseguridad de las empresas a nivel nacional y local, las perdidas por victimización, los entornos inmediatos en los que operan las empresas y los fallos en materia de seguridad pública.

Desde la teoría del lugar peligroso, como su nombre lo indica, el lugar en donde se ubican las empresas es determinante de la percepción de inseguridad y del miedo al delito. Así, se establece una relación positiva en donde al aumentar la percepción de riesgo o peligro respecto al lugar, se incrementa la prevalencia a adquirir e instalar tecnologías de vigilancia como medida de protección.

Sin embargo, también se advierte que hay determinadas conductas delictivas o antisociales que en mayor medida se asocian con la disposición a invertir en este tipo de tecnologías, como la extorsión o cobro de piso que afecta directamente a los establecimientos y violentan la vida de quienes ahí trabajan.

Paralelamente, en esta investigación se identificaron distintas formas de complementariedad de la videovigilancia. Por una parte, se encontró que las empresas implementan de forma complementaria otras medidas de protección, y sobresale la adquisición de diferentes tecnologías como los dispositivos de localización con GPS.

Asimismo, se observó que un porcentaje de empresas que han instalado cámaras privadas refieren que no tienen conocimiento de la instalación de videocámaras de vigilancia públicas por parte de los gobiernos locales, y ante mayor desconocimiento de este tipo de acciones por parte de las autoridades, incrementa la inversión en tecnologías de vigilancia privadas. Sin embargo, también se advierte que existen empresas que aún y cuando reconocen la instalación de cámaras de vigilancia públicas, deciden invertir en sus propios sistemas de vigilancia. Probablemente como una forma complementaria o como una manifestación social de desconfianza o disputa por la vigilancia en sus propios entornos.

En la lógica de la complementariedad de la vigilancia pública y privada se encontró evidencia de esquemas de "videovigilancia colaborativa" en donde las empresas han logrado conectar sus 
sistemas de alarmas y cámaras de videovigilancia con los centros de monitoreo públicos para acceder de manera inmediata a la atención de los delitos y emergencias. Esta nueva forma de colaboración muestra que la prevención no es un asunto exclusivo de las empresas y también se promueve desde los gobiernos a través de la política pública.

$\mathrm{Al}$ respecto es importante enfatizar que la política pública de videovigilancia, debe contemplar que la atención directa a las empresas no sea en detrimento de la atención a la población que también tiene derecho a ser atendido prioritariamente ante un delito o una emergencia.

Finalmente, solo quisiera apuntar la relevancia de la investigación sobre las empresas a través de instrumentos como la ENVE que aquí se ha utilizado, y cuyos resultados pueden ser puntero para la generación de hallazgos relacionados con la teoría de las actividades rutinarias, la teoría de la elección racional, la teoría del lugar peligroso, entre otras. Así como también, ser la base para la elaboración de políticas públicas en la materia.

\section{Referencias}

Becker, G. (1968). Crime and Punishment: An Economic Approach. En The economic dimensions of crime (pp. 13-68). London: Palgrave Macmillan.

Bressler, M. (2009). The impact of crime on business: A model for prevention, detection \& remedy. Journal of management and Marketing Research 2(1), 12-20.

Cervini, R. (2007, Noviembre). Abordaje metodológico de la delincuencia económica sofisticada. Conferencia dictada en el Curso de Especialización en Derecho Penal Económico, Brasil: Facultad de Derecho, Universidad Getulio Vargas de San Pablo, 1-44

Correa, G. (2013). Desarrollo Empresarial, Inversión Extranjera y Crimen Organizado en México: Los Efectos Reales de la Violencia (2006-2010). Panorama Socioeconómico 31(46), 29-40.

Cosme, M. (2019, 28 de agosto). Ofrecen posiciones a empresarios en el C5 para combatir el crimen, El Sol de México. Recuperado de: https://www.elsoldemexico.com.mx/metropoli/policia/ofrecenposiciones-a-empresarios-en-el-c5-para-combatir-el-crimen-4100660.html

Excélsior (2012, 3 de abril). Más de 160 mil empresas cierran por inseguridad, afirma la Coparmex. Excélsior. Recuperado de: https://www.excelsior.com.mx/2012/04/03/nacional/823682

Figueroa, L., Montes de Oca, R., Rivera, L., Artavia, R., \& Marshall, L. (1997). Impacto de la Inseguridad Pública sobre el Clima de Negocios e Inversión de Centroamérica. En Centro Latinoamericano de Competitividady Desarrollo Sostenible del INCAE 80, 1-74.

Heraldo (2018, 27 de noviembre). 58\% de las empresas de Coparmex, víctimas de inseguridad, afirman. Heraldo de México. Recuperado de: https://heraldodemexico.com.mx/mer-k-2/58-de-las-empresas-decoparmex-victimas-de-inseguridad-afirman/

INCIBE (2016). Tecnologias biométricas aplicadas a la ciberseguridad. Una guia de aproximación para el empresario. España: Instituto Nacional de Ciberseguridad (INCIBE).

INEGI (2018). Encuesta Nacional de Victimización y Percepción sobre Seguridad Pública 2018 (ENVIPE). Principales Resultados. México: INEGI.

INEGI (2018a). Encuesta Nacional de Victimización de Empresas 2018 (ENVE). Principales Resultados, México: INEGI.

Jasso C. (2019). Prevención del delito y tecnología: La instalación de cámaras de videovigilancia y alarmas como medida de protección de los hogares en México. Estudios de la Seguridad Ciudadana, 2(2), 159186.

Hipertextos, Vol. 8, N 14. Buenos Aires, Julio/Diciembre de 2020 «109 DOI: https://doi.org/10.24215/23143924e021, https://revistas.unlp.edu.ar/hipertextos 
Jasso C. (2013). Percepción de inseguridad en México. Revista Mexicana de Opinión Pública, 15, 12-29. Nader, S. (2019, 4 de junio). Código C5 permitirá alertar para proteger negocios. El Valle. Recuperado de: https://www.elvalle.com.mx/estado-de-mexico/story/2940/codigo-c5-permitiraalertar-para-proteger-negocios

Orquiz, M. (2019, 27 de noviembre). Videovigilancia ciudadana: otra mirada contra la delincuencia. El Diario. Recuperado de: https://laverdadjuarez.com/index.php/2019/11/28/videovigilanciaciudadana-otra-mirada-contra-la-delincuencia/

Ramírez de Garay, L. (2014). Crimen y economía: una revisión crítica de las explicaciones económicas del crimen. Argumentos 27(74), 261-290.

Rojas, F. (2007). Mayor presencia del crimen organizado: Consecuencias de la crisis de gobernabilidad y el débil imperio de la ley. Foreign affairs: Latinoamérica, 7(2), 22-35.

Rubio, M. (1998). La economía en una sociedad violenta. Revista de Estudios Sociales, (1), 22-32.

Sanllehí, A. (2009). Prevención del Delito en la empresa. Revista Electrónica de Ciencia Penal y Criminología, 11 (10), 1-48. Recuperado de: http://repositori.uic.es/handle/20.500.12328/1461.

Soria, R. (2017). El impacto de la inseguridad pública en la competitividad empresarial: análisis comparativo de las entidades federativas en México. Economía y Sociedad, 21(36), 19-41.

Solís, G. (2007). Pandillas juveniles y gobernabilidad democrática en América Latina y el Caribe. Memorias del seminario internacional Madrid, 16.

Stuart, M. (2014). Importancia de medir la delincuencia contra el sector privado para diseñar políticas públicas que prevengan y combatan este fenómeno: algunas de las mejores prácticas. En Mugellini, Giulia (Coordinadora) (2014), Medición y análisis de la delincuencia contra el sector privado: experiencias internacionales y el caso mexicano, México: INEGI.

Tello, J. (2007). Reflexiones sobre el impacto de la inseguridad en las empresas. Foreign affairs: Latinoamérica, 7(2).

Vilalta, C. (2018). La victimización de las empresas en México: conceptos, teorías y mediciones. Gestión y Politica Pública, XXVII, (2), 501-540.

Vilalta, C. (2017). Cuando la cleptocracia no alcanza: los delitos contra las empresas. Economía, sociedad y territorio, 17 (55), 837-866.

Zevallos, E. (2003). Micro, pequeñas y medianas empresas en América Latina. Revista de la CEPAL. 79, 53-70.

Hipertextos, Vol. 8, N 14. Buenos Aires, Julio/Diciembre de 2020 «110 DOI: https://doi.org/10.24215/23143924e021, https://revistas.unlp.edu.ar/hipertextos 